\title{
Low-order wavefront sensing in tomographic multi-beacon adaptive optics systems
}

Viswa Velur, Ralf Flicker, Richard Dekany, Gustavo Rahmer, Roger Smith, et al.

Viswa Velur, Ralf Flicker, Richard Dekany, Gustavo Rahmer, Roger Smith, Anna Moore, "Low-order wavefront sensing in tomographic multi-beacon adaptive optics systems," Proc. SPIE 7015, Adaptive Optics Systems, 70154W (14 July 2008); doi: 10.1117/12.788019

Event: SPIE Astronomical Telescopes + Instrumentation, 2008, Marseille, France 


\title{
Low-order wavefront sensing in tomographic multi-beacon adaptive optics systems
}

\author{
Viswa Velur ${ }^{a}$, Ralf Flicker ${ }^{b}$, Richard Dekany $^{a}$, Gustavo Rahmer ${ }^{a}$, Roger $\operatorname{Smith}^{a}$, Anna Moore $^{a}$ \\ ${ }^{a}$ Caltech Optical Observatories, California Institute of Technology, Pasadena, CA 91125, US \\ ${ }^{b}$ W. M. Keck Observatory, Kamuela, HI 96743, USA
}

\begin{abstract}
We present a concept to perform low-order wavefront sensing in multi-laser guide star adaptive optics systems operating using a large format NIR detector with windowing capability with near diffraction limited or partially corrected NGS tip-tilt stars with time varying Strehls. Most contemporary adaptive optics systems in development for large telescopes, viz., the next VLT adaptive optics facility that serves as a pathfinder to the European ELT, Gemini MCAO, W. M. Keck observatory's Next Generation Adaptive Optics (NGAO) System, The Large Binocular Telescope and the Thirty Meter Telescope's NFIRAOS are multi-laser guide star systems that provide $\mathrm{AO}$ correction over a large field. In such systems even faint tip-tilt (TT) stars image are characterized by either a well corrected (MOAO case) or at least a partially corrected (MCAO or GLAO case) diffraction limited core due to high order sharpening by the LGS WFS. In such a regime of low-order sensing one could envisage using pixels as field stops and choosing a appropriate plate scale to minimize the sky background.

Simulations are used to predict the performance of such a sensor when guiding on point sources and on extended objects of varying brightness and for different levels of high order correction.

The parameter space explored includes tip-tilt and tip-tilt, focus and astigmatism (TTFA) sensor performance for various plate scales, TT sensor performance vs. level of high order correction (TT star Strehl) and TT sensor performance vs. TT object size for a given detector noise, gain and a simple centroiding algorithm. Due to small sky noise contribution at plate-scales le $100 \mathrm{mas} / \mathrm{pixel}$, the optimum low-order wavefront sensor plate scale is found to be 80-100 mas/pixel $(3 \times-4 \times \lambda / \mathrm{d}$ in J- and H- bands) for the Keck NGAO system.
\end{abstract}

Keywords: Multiple laser guide star AO, Low-order sensing, adaptive optics

\section{INTRODUCTION}

The evolution of laser guide star adaptive optics and the maturity in laser technology have culminated in the sky coverage being restricted to finding and obtaining low-order wavefront information from natural TT(FA) guide stars in future facility-class adaptive optics systems. In this case it is vital to be able to acquire and guide on faint natural stars to obtain tip-tilt information. We present a technique to acquire and guide on both point sources and extended objects ${ }^{10}$ like comets, with an IR detector with a $\sim 100$ mas/pixel detector plate scale. We predict performance of such AO systems using simulations in this paper. Practical implications of this concept including dithering, tracking non-sidereal tracking and differential atmospheric refraction may also influence the final decision on the sensor design and plate scale. These effects will be included in the model and dealt with in detail in the next two phases of the NGAO project.

The architecture for the sensor described in this paper has been used as baseline for the W. M. Keck Observatory's NGAO concept study ${ }^{1}$ and we are actively involved in prototyping the concept presented. The concept can be extended to other multi-guide star AO systems using tools we have developed.

Author to whom correspondence should be sent:

Viswa Velur Robinson 01, M/C - 105-24, 1200 E. California Blvd., Pasadena, CA - 91125. E-mail: vnv@phobos.caltech.edu.

Adaptive Optics Systems, edited by Norbert Hubin, Claire E. Max, Peter L. Wizinowich,

Proc. of SPIE Vol. 7015, 70154W, (2008)

0277-786X/08/\$18 doi: $10.1117 / 12.788019$

Proc. of SPIE Vol. $701570154 \mathrm{~W}-1$ 


\subsection{Overview of NGAO Wavefront Sensors}

NGAO has nine Laser Guide Star (LGS) wavefront sensors, two NGS tip-tilt sensors, one NGS tip-tilt, focus and astigmatism sensor and dedicated widefield and narrow field truth or calibration wavefront sensors ${ }^{2}{ }^{3}{ }^{4}$ All of the wavefront sensors can patrol within their field of regard to pick off stars. The LGS wavefront sensors provide high order wavefront information at high frame rates, while the low-order wavefront sensors provide low order wavefront information that is either not sensed or not sensed well by the LGS sensors at atmospheric time scales. The truth sensor (TWFS) (synonymous with the calibration wavefront sensor) is used to calibrate biases that arise when using LGS in an adaptive optics system. ${ }^{5}$ The biases are principally caused by the elongated nature of the LGS when viewed by sub-apertures of the laser guide star wavefront sensor and the changing sodium layer density profile. The truth wavefront sensor measures slowly varying biases of low spatial order by sensing the wavefront from a point source (a natural star). A natural guide star WFS using long exposures and only measuring the lowest spatial wavefront error is sufficient. The truth sensor could potentially also be used for tracking null and blind modes beyond the measurement capability of the low order wavefront sensor (LOWFS) if read at atmospheric time scales with larger number of sub-apertures.

\section{LOW ORDER WAVEFRONT SENSORS}

LOWFS use natural stars to sense low order modes of the wavefront that are poorly sensed by the multiple LGS. ${ }^{11}$ These modes include tip and tilt, which are naturally filtered out of LGS wavefront measurements and higher order modes that are invisible to or partially sensed by the Laser Guide Star wavefront sensors. ${ }^{6}$ Low order wavefront sensors sense tip, tilt, focus and astigmatism when the goal is optimizing on-axis science performance. When the goal is optimizing science performance averaged over a larger field of view the LOWFS is used to estimate tip, tilt and tilt anisoplanatism modes. In both cases these sensors account for the vertical motion of the sodium layer. Based on trade studies conducted by the NGAO team, ${ }^{12}{ }^{15}$ two IR quad-cell tip-tilt sensors and one $2 \times 2$ sub-aperture IR Shack-Hartmann (SH) based TTFA sensor have been posited to be sufficient for NGAO and TMT. In order to maximize sky coverage, the IR LOWFS will work in J- and H-bands simultaneously. At these wavelengths, we envisage reading the detector multiple times non-destructively to reduce the read noise. ${ }^{7}$ The NGAO conceptual design uses a dedicated AO relay for each of the 3 low-order wavefront sensors. In the NGAO case, better TT performance can be obtained when guiding on the core of a PSF at H-band Strehls greater than $12 \%$ under median Mauna Kea atmospheric conditions as compared to guiding on the halo around the core.

Schematics in figures 1 and 2 depict the concept. Figure 1 shows a TTFA sensor with a cold front end and a cryogenic back end with both the Deformable Mirror (DM) and the Shack Hartmann (SH) lenslet array at pupils. A variant that has better transmission has also been considered where the DM is at $20 \mathrm{~km}$ conjugate and the lenslet at a pupil. The propagation effects due to scintillation will be studied for this configuration in the preliminary design phase of NGAO. To minimize background emissivity of optics, whole AO system is cooled to $\sim-20^{\circ} C^{13}$ and the MEMS DM and the high speed tip-tilt stage on which the DM is mounted are kept outside the cryogenic chamber so as to minimize cost of the mechanisms. To minimize system complexity and NGS guide star acquisition time, the TWFS is feed off of a small fraction of light from the TTFA sensor that nominally guides on the brightest star in the field. The lenslet array splits the light at the pupil to form a $2 \times 2 \mathrm{SH}$ spots based on a trade study. ${ }^{8}$ One of the lenses that is required for the system is also used as the vacuum window for the cryogenic chamber. Figure 2 shows the schematic of the TT sensor, this sensor is similar to the TTFA except for the fact that it needs only one pupil, where the DM is located and it has a planar vacuum window.

\section{SIMULATIONS}

Based on work done for $\mathrm{NGAO}^{4} 89$ the authors simulated different architectures for low order wavefront sensing. The main conclusions from these studies were that the pyramid sensor is only marginally better than a $2 \times 2 \mathrm{SH}$ sensor for tip-tilt sensing; and high order sharpening seems to have a dramatic effect on the performance of the sensor. Based on these two results we chose to baseline our design with an IR SH sensor (to do away with the issue of obtaining a high quality pyramid and modulating the glass pyramid in a cryogenic chamber). Further simulation work was carried out based on the results from the low order wavefront sensor architecture trade 


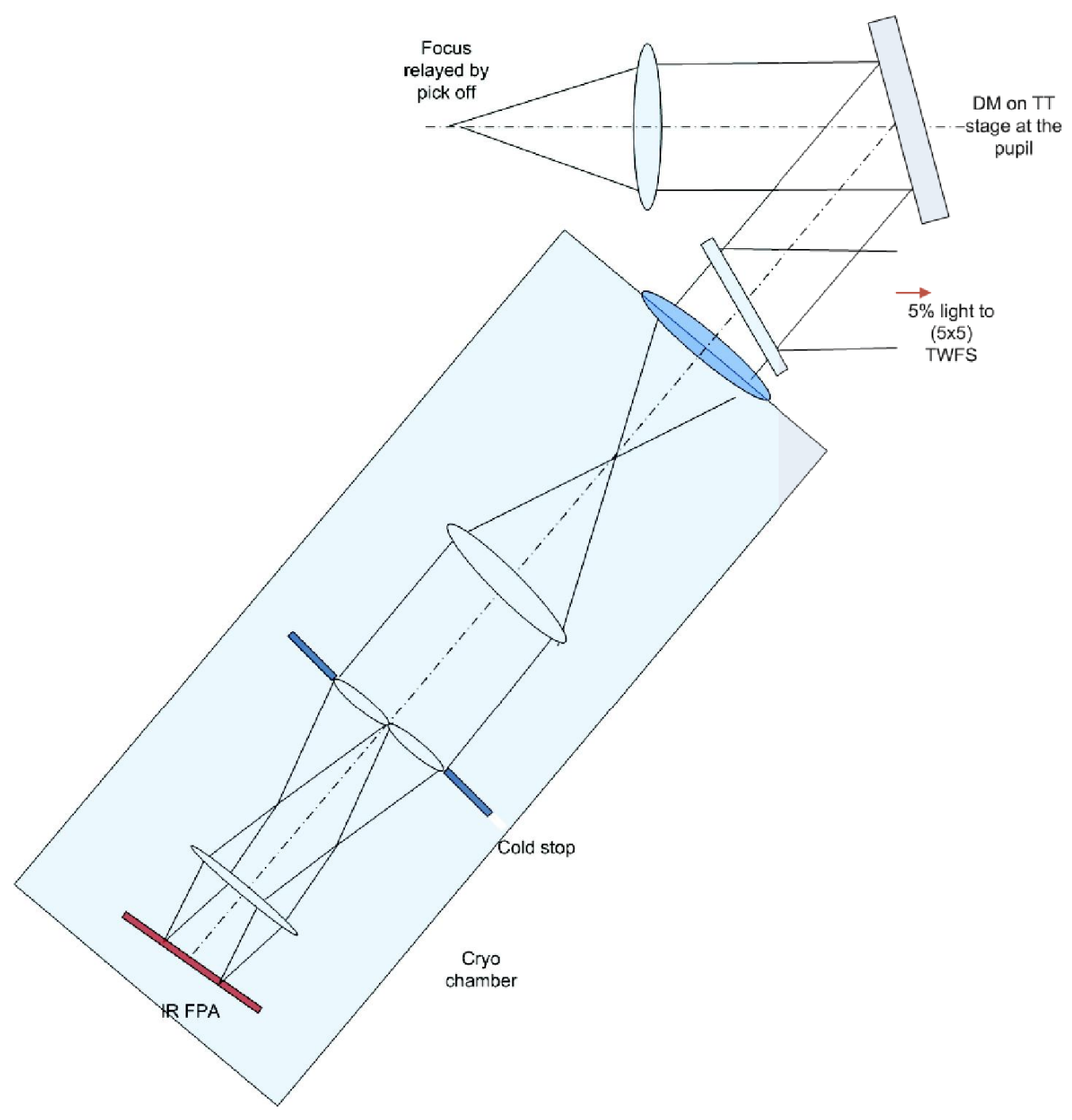

Figure 1. Schematic of the Keck NGAO TTFA sensor showing the cold stop and cryogenic part and the $\left(-20^{\circ} \mathrm{C}\right)$ front end (there is a window between the cold and the cryogenic chamber). TWFS - Truth wavefront sensor, IR FPA - Infra-red Focal Plane Array. 


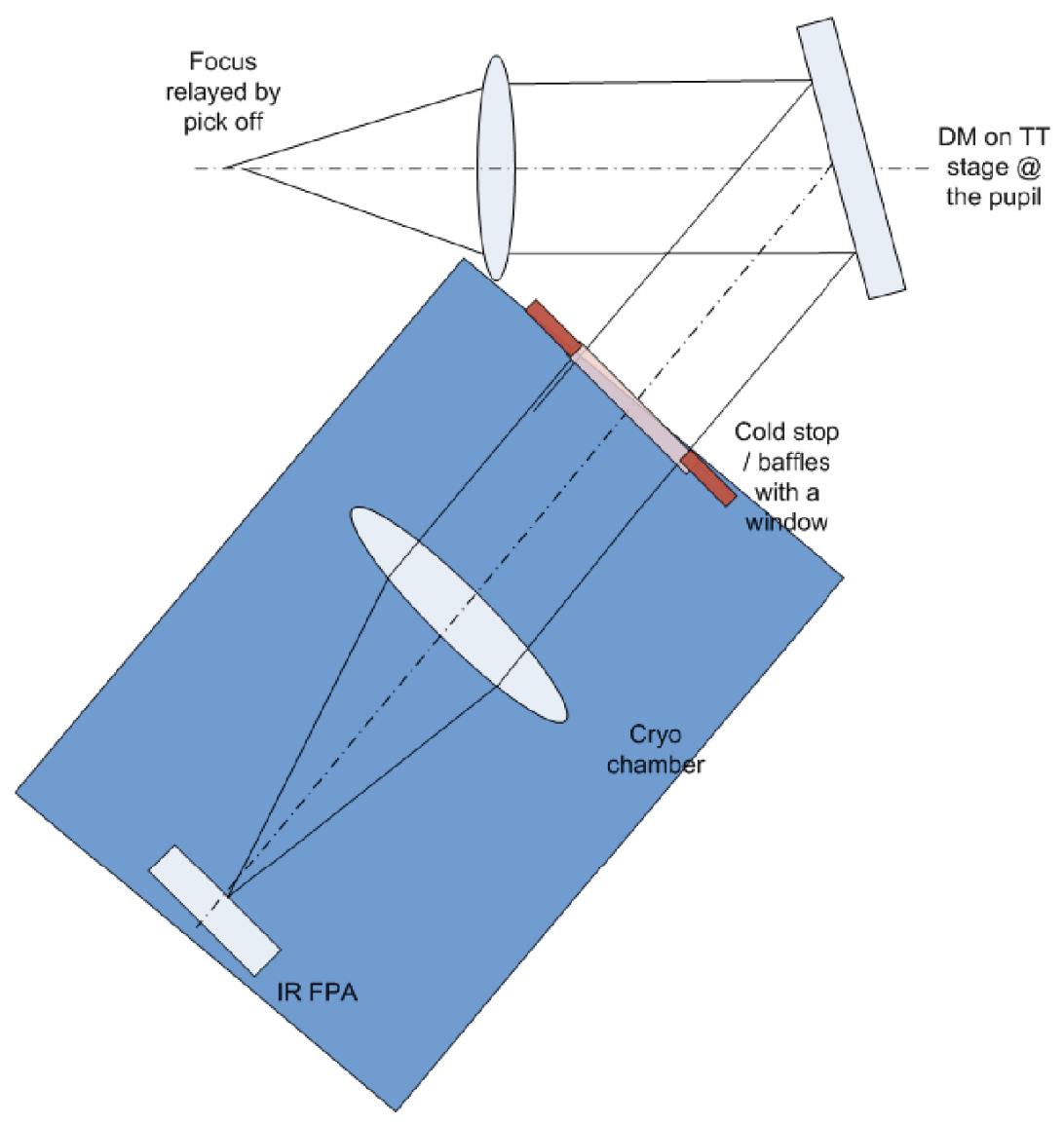

Figure 2. Schematic of the TT sensor design (there is a window between the cold and the cryogenic chamber).

\begin{tabular}{lrl}
\hline \hline Turbulence model & 7-layer CN-M3 & KAON 303 \\
Fractional $C_{n}^{2}$ & {$[0.471,0.184,0.107,0.085,0.038,0.093,0.023]$} & units of $\mu_{0}$ \\
Altitudes & {$[0.0,2.1,4.1,6.5,9.0,12.0,14.8]$} & $\mathrm{km}$ \\
Wind speeds & {$[6.7,13.9,20.8,29.0,20.0,20.0,20.0]$} & $\mathrm{m} / \mathrm{s}$ \\
$r_{0}($ at $0.5 \mu \mathrm{m})$ & 18 & $\mathrm{~cm}$ \\
$d_{0}($ at $0.5 \mu \mathrm{m})$ & 4.4 & $\mathrm{~m}$ \\
$\tau_{0}($ at $0.5 \mu \mathrm{m})$ & 3.8 & $\mathrm{~ms}$ \\
Outer scale $L_{0}$ & 30 & $\mathrm{~m}$ \\
Zenith angle & 0 & \\
\hline \hline
\end{tabular}

Table 1. Turbulence parameters for LOWFS simulations.

study $^{8}$ and this work is summarized as an appendix in the NGAO WFS conceptual study report. ${ }^{4}$ The new simulation codeis capable of performance prediction with extended sources with variable detector read out region by implementing a variable centroiding algorithm. A method of capturing a wandering core was also simulated. Tables 1 and 2 indicate the turbulence parameters and the photometry used for the simulations. Figure 3 shows a typical simulation result.

\subsection{Extended sources}

A simple model for extended reference sources was implemented using a flux-preserving convolution with a uniform disk of a given diameter. In incoherent imaging, the image in the far field can be described by the PSF 


\begin{tabular}{cccccc}
\hline \hline $\begin{array}{c}\text { Wavelength } \\
\text { Band }\end{array}$ & $\begin{array}{c}\text { central } \lambda \\
(\mu \mathrm{m})\end{array}$ & $\begin{array}{c}\text { FWHM } \\
(\mu \mathrm{m})\end{array}$ & $\begin{array}{c}\text { color } \\
\text { correction }\end{array}$ & $\begin{array}{c}\text { zero point flux } \\
\left(10^{10} \text { photons } / \mathrm{s}\right)\end{array}$ & $\begin{array}{c}\text { sky brightness } \\
\left(\mathrm{mag} / \operatorname{arcs}^{2}\right)\end{array}$ \\
\hline $\mathrm{U}$ & 0.366 & 0.052 & -0.2 & 19.6 & 20.90 \\
$\mathrm{~B}$ & 0.438 & 0.100 & -0.6 & 68.4 & 22.13 \\
$\mathrm{~V}$ & 0.545 & 0.083 & 0.0 & 40.6 & 21.99 \\
$\mathrm{R}$ & 0.641 & 0.157 & 0.6 & 54.1 & 20.81 \\
$\mathrm{I}$ & 0.798 & 0.154 & 1.3 & 34.2 & 20.25 \\
$\mathrm{~J}$ & 1.22 & 0.260 & 2.7 & 26.3 & 19.60 \\
$\mathrm{H}$ & 1.63 & 0.290 & 3.8 & 14.2 & 17.09 \\
$\mathrm{~K}^{\prime}$ & 2.12 & 0.410 & 4.9 & 9.39 & 16.99 \\
$\mathrm{~K}$ & 2.19 & 0.320 & 4.8 & 8.02 & 16.78 \\
$\mathrm{~L}$ & 3.45 & 0.570 & 6.8 & 3.67 & 9.91 \\
\hline \hline
\end{tabular}

Table 2. Photometric system based on the Johnson-Cousins-Glass system from Bessel et al. (1998). The zero point was computed for a collecting area of $79 \mathrm{~m}^{2}$, and is the photon flux at the Keck Nasmyth from a $M_{v}=0$ star.

of the imaging system convolved with the source intensity distribution. This was implemented in the LOWFS simulation code by multiplying the instantaneous speckle OTF in each sub-aperture with a jinc function, being the Fourier transform of a disk: $O T F_{\text {ext }}(\rho / \lambda)=2 J_{1}(\theta \pi \rho / \lambda) /(\theta \pi \rho / \lambda)$. Where $\theta$ is the radius of the disk, $\lambda$ is the wavelength, and $\rho$ is the radial spatial variable.

\subsection{Variable detector read-out zone}

In our simulations, instead of designing the LOWFS sub-aperture detectors with a fixed read-out zone, the number of pixels in a square box can be varied. The idea is that this will allow one to design a smaller plate scale than traditionally for Shack-Hartmann sensors, which will take better advantage of the partially corrected spot provided by the high-order AO system. For a simulation where the pixel size is 50 mas, and the the initial (maximum) read-out region is a $8 \times 8$ pixels and the numbers of pixels used for centroiding is reduced as the spot is captured by the sensor. The fact that the plate scale is small makes the sky noise negligible, and the performance is entirely read-noise limited. Initially the LOWFS detector read-out zone has to be large, while the high-order system is closing the loop. After convergence, the LOWFS detector read-out region can be shrunk down to increase sensitivity and SNR. In the case of extended reference sources, the read-out zone can be adjusted to match the size of the object in order to optimize Signal-to-Noise Ratio (SNR).

\subsection{Variable centroiding algorithm}

In addition to varying the read-out zone, the centroiding method can also be adjusted in real-time. When the read-out zone is larger than $2 \times 2$ pixels, one can choose between a weighted Center Of Gravity (COG) calculation or the simple quad-cell formula (for $2 \times 2$ pixel read-out they are equivalent). The quad-cell formula bins the quadrants in software if there is no on-chip binning capability. This means experiencing the full contribution of read-noise in each pixel. For extended sources and large read-out zones, however, it seems likely (to be verified at a later stage) that the quad-cell formula will work better since it does not amplify read-noise along the perimeter of the detector region, but instead averages the read-noise from all pixels in a quadrant evenly weighted. The purpose of using the weighted COG formula is to extend the linear response of the sub-aperture, a function which only is valuable when the excursions are large and the noise in the outer rings of the detector read-out zone is small. A typical H2RG detector with 32 readout channels can read out four sets of $10 \times 10$ pixels at atmospheric time scales $(\sim 200 \mathrm{~Hz}-400 \mathrm{~Hz})$ with Fowler sampled read noise of less than $7 e^{-}$s. The code can accommodate other centroiding algorithms as well.

\subsection{Conclusions from recent simulations}

It is apparent from the plots on figure 4 that for bright point sources the exact plate scales are not vital. While for fainter stars $\left(>16 M_{v}\right.$ ) both the 40 mas/pixel (smallest plate scale) and 200 mas/ pixel (the largest plate scale) seem to perform worse than intermidate plate scales. The reason for this effect is that at 200 mas/pixel 


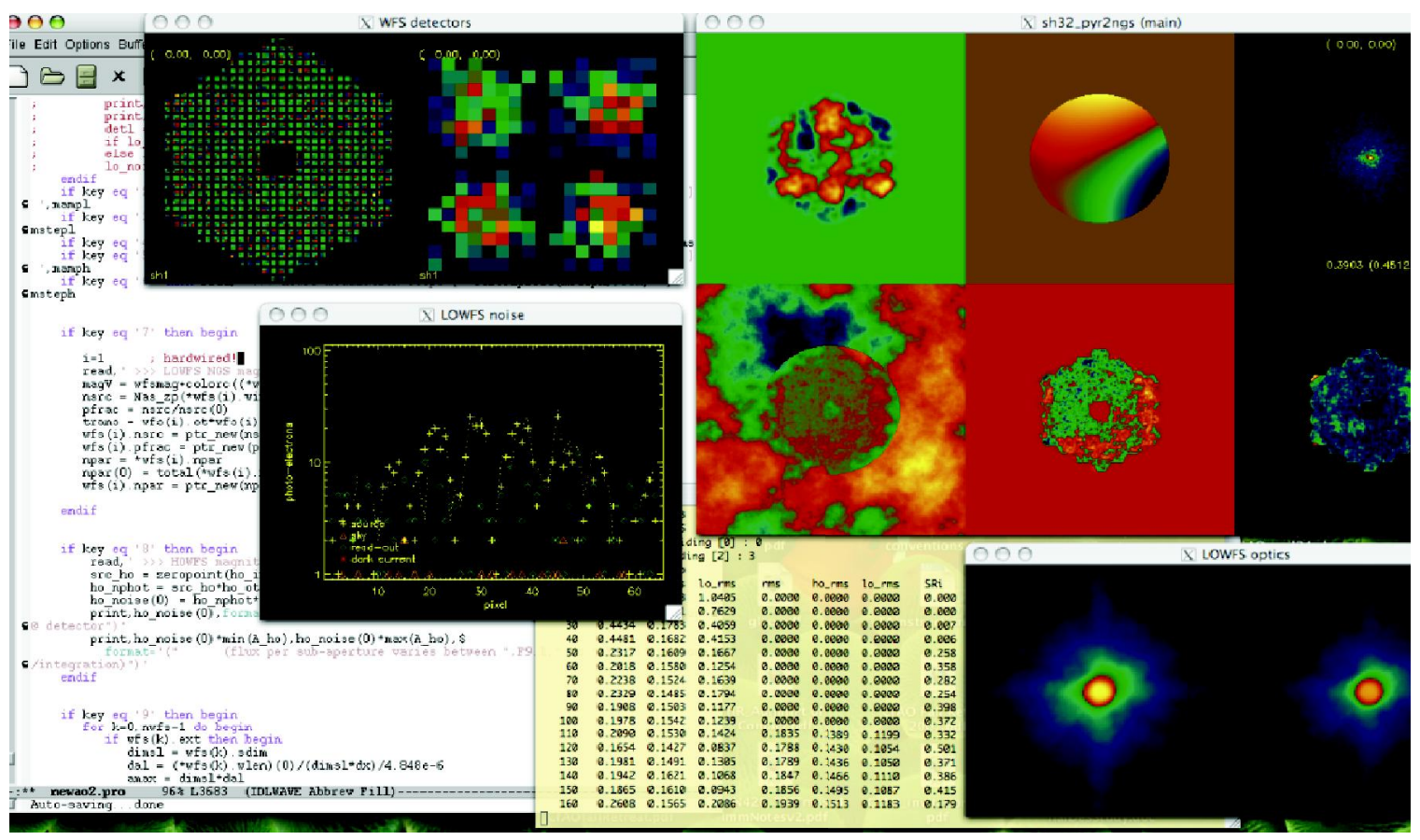

Figure 3. Sample simulation of a TT sensor with 200 mas size object and 30 mas/ pixel scale for the Keck NGAO case.
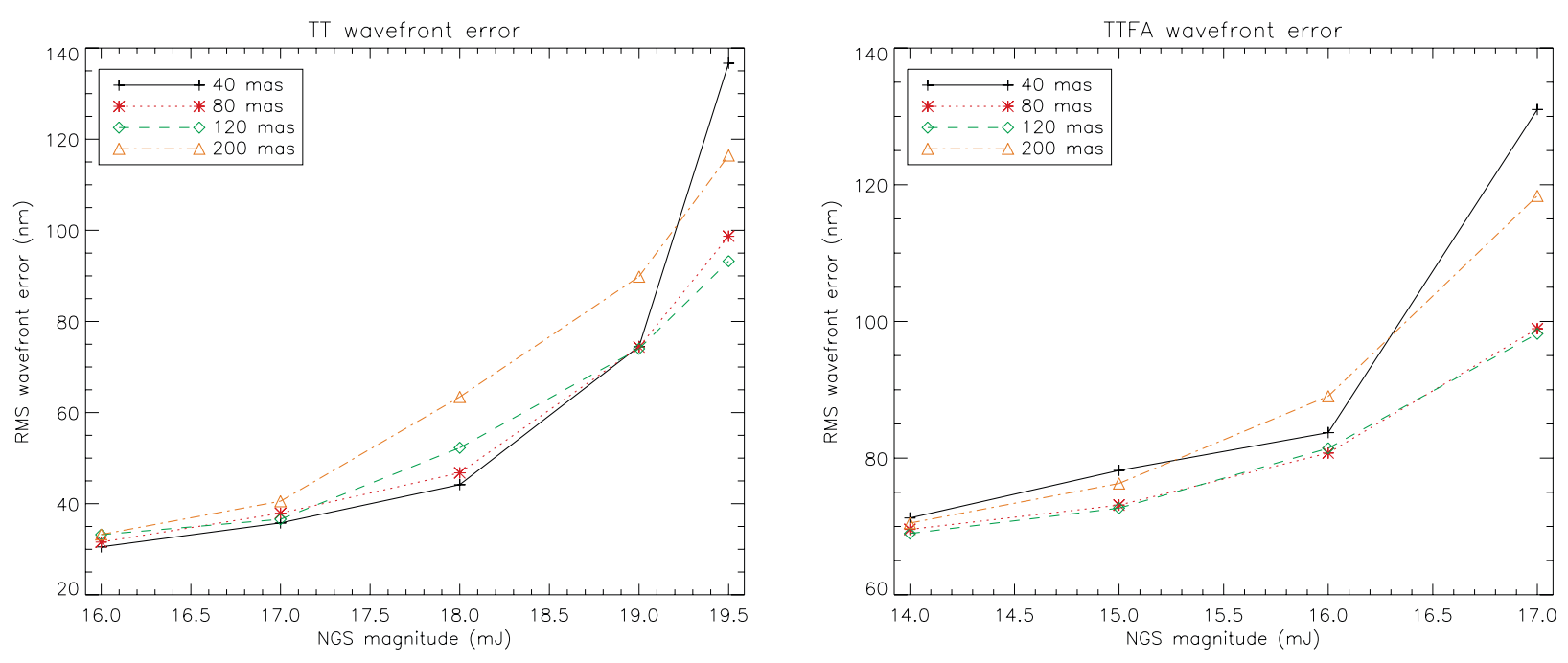

Figure 4. LOWFS performance versus NGS J magnitude and detector plate scale, for a TT sensor correcting only two Zernike modes (left) and a TTFA sensor correcting the first five Zernike modes (right), with a high-order RMS wavefront error of $185 \mathrm{~nm}$ in the incident LOWFS beam.

the sky noise starts to affect the SNR and at 40 mas/pixel the spot excursions become too large, this leads to loop instability which forces one to use more pixels to centroid with additional read noise. Based on the above plots, a plate scale of 80 to $100 \mathrm{mas} / \mathrm{pixel}$ is an ideal for this sensor for the NGAO case.

Figure 5 shows how the wavefront error vs. angular size of the guide star and level of high order correction for a $M_{v}=17$ star with the plate scale chosen choice of 100 mas/pixel. 

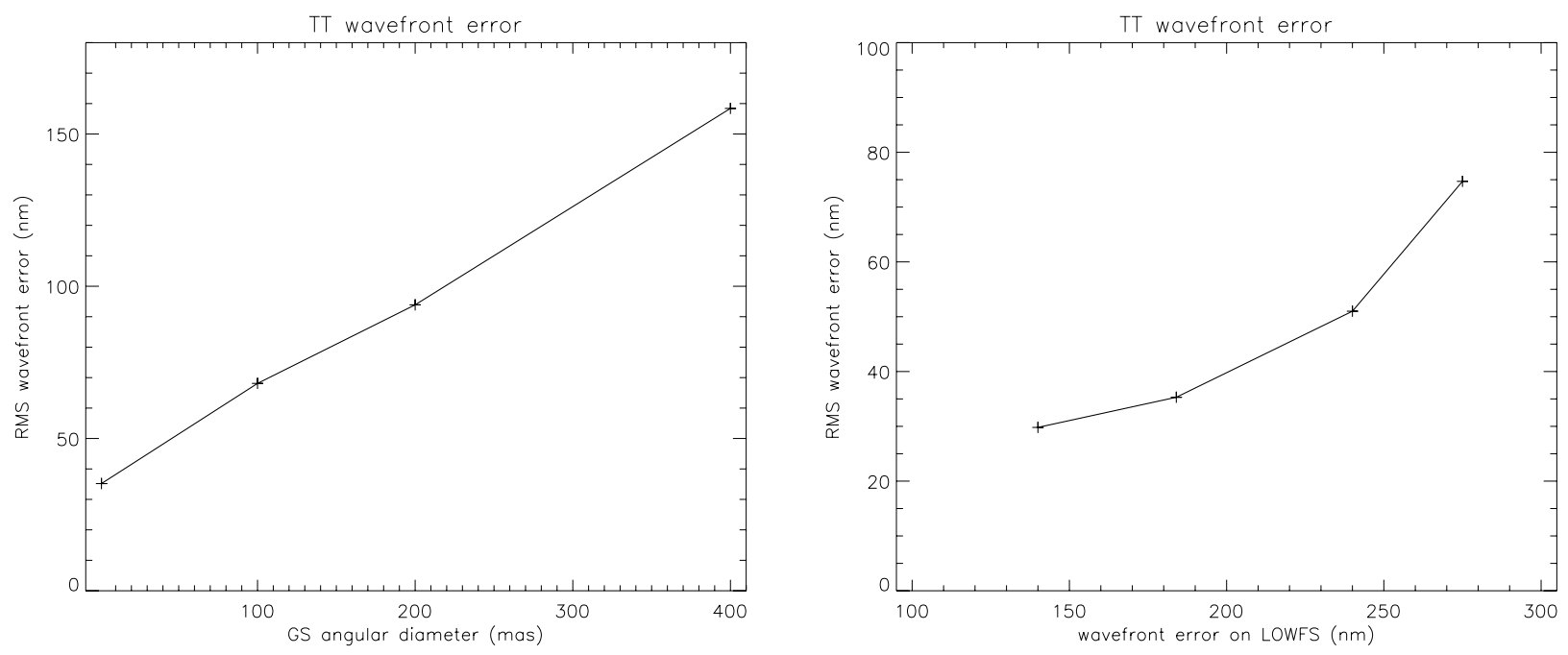

Figure 5. Residual TT waveront error versus guide star angular diameter for a $M_{J}=17$ source (left) and high-order wavefront error incident on the LOWFS (right).

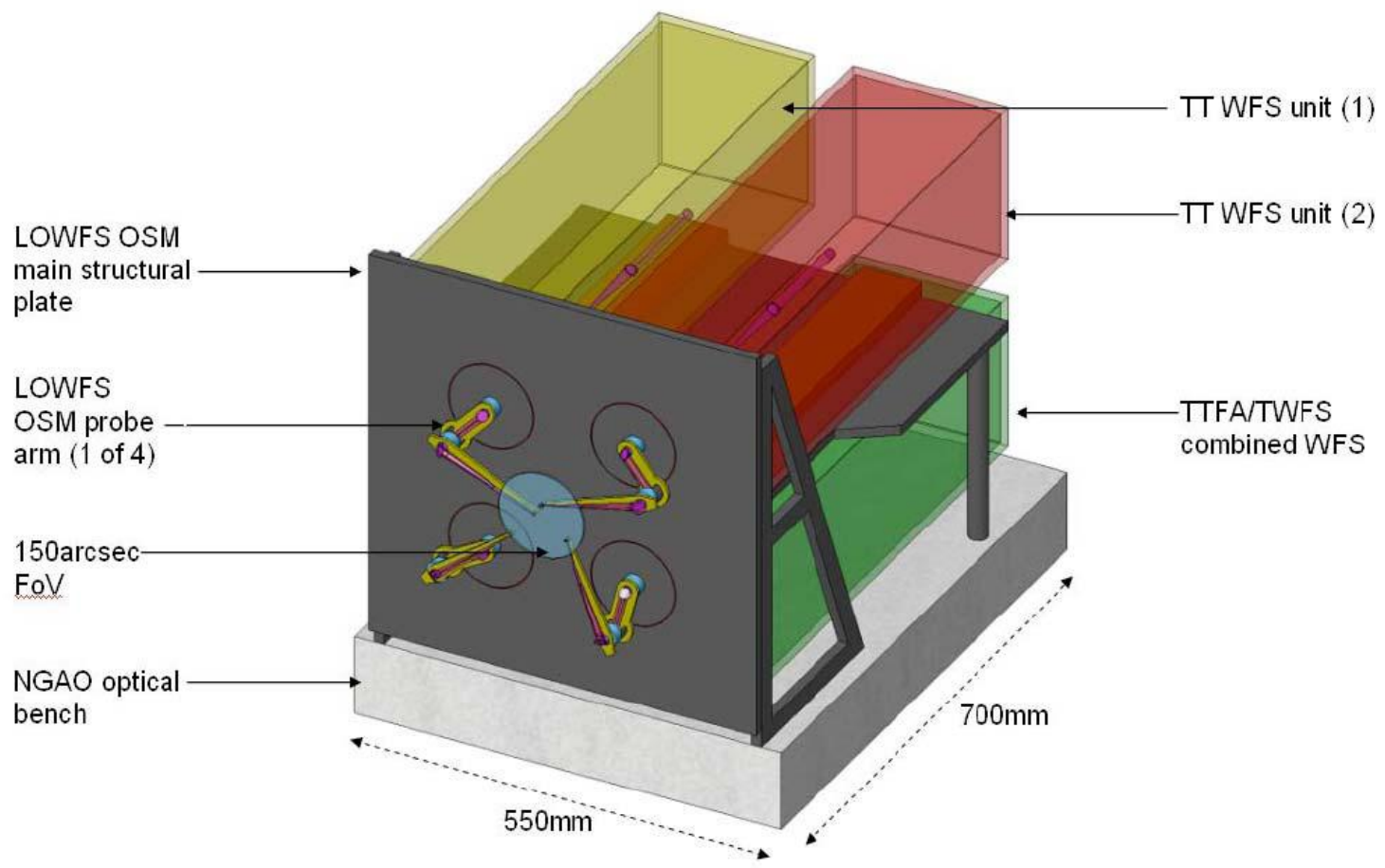

Figure 6. Schematic of the TT sensor design (there is a window between the cold and the cryogenic chamber).

\section{NOISE MEASUREMENTS FROM A NIR LARGE FORMAT DETECTOR}

To simulate the noise performance of a real detector working as a WFS, we devised an experiment using a 2.5 um cut-off Hawaii-2RG detector (cooled at $80 \mathrm{~K}$ ) operating in 32-channel mode, where a single pixel per channel was sampled 131,000 times at a rate of $100 \mathrm{KHz}(10 \mu \mathrm{sec}$ per sample), without resetting or clocking the array, resulting in a one-dimensional vector of 16-bit values per channel. Every vector was then processed 


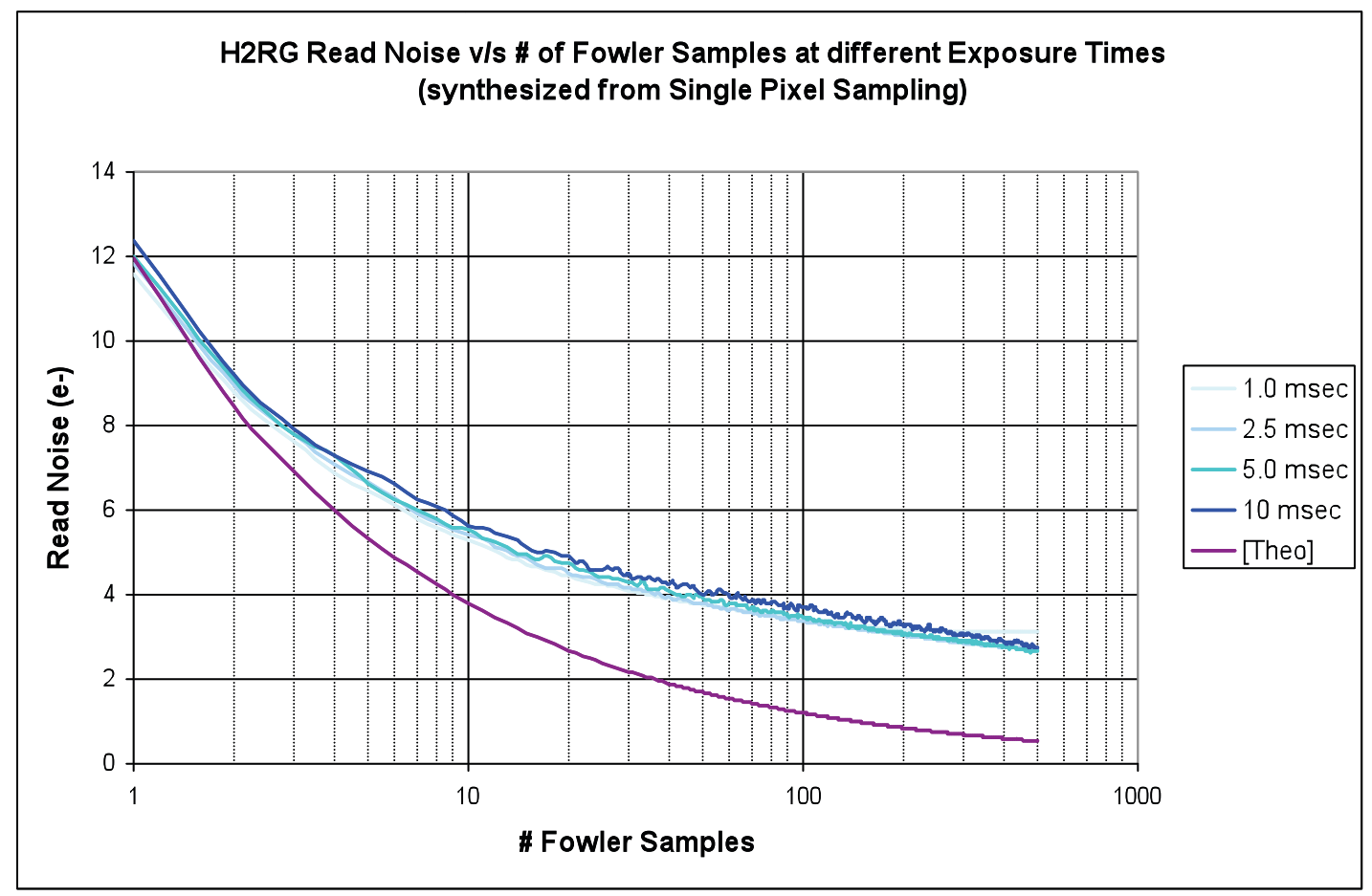

Figure 7. Hawaii $2 \mathrm{RG}$ measurements - 131,000 samples were acquired at $100 \mathrm{KHz}$ on each of the 32 channels without resetting or clocking. Sequential (non-overlapping) Fowler sampled integration were synthesized from the data stream at various depths to simulate lower pixel visitation rate due to Region-Of-Interest (ROI) readout through a single channel, only every N-th sample.

using MATLAB to produce sequential (non-overlapping) synthesized pixel values for different numbers of Fowler samples (1 to 500) and different exposure times (1.0, 2.5, 5.0 and $10.0 \mathrm{msec})$. We envision using this NIR detector for our low-order wavefront sensors.

Fowler sampling ${ }^{17}$ is a readout method used in IR arrays to reduce read noise by performing multiple nondestructive reads at the beginning and the end of the integration. The average for each group of samples is computed and the results are subtracted to obtain the final pixel value. The exposure time is defined as the time between the first sample of the first group, and the first sample of the second group. With this definition, the minimum exposure time is determined by the number of samples. The read noise was calculated as the standard deviation of the pixel values for every combination of number of Fowler samples and exposure times. The results are shown in Fig. 7. The theoretical curve, where the noise reduction is proportional to $\frac{1}{\sqrt{N}}$, is included for reference (using an average value for the read noise at $\mathrm{N}=1$ ).

As indicated by the graph, the read noise depends not only on the number of Fowler samples, but, also upon the exposure time, due to $\frac{1}{f}$ noise. Note that the higher the exposure time, the higher the noise. For all exposure times, the read noise drops below $6 \mathrm{e}-$ for $\mathrm{N} \geq 10$.

\section{PROTOTYPING EFFORT}

To meet performance goals for NGAO, we plan to prototype this sensor as part of the NGAO preliminary design phase. The top level goal of the LOWFS assembly prototyping is to produce a single TT WFS unit with a beam feeding probe arm that as a whole satisfies the functional requirements. We can simulate the fully or partially corrected beam using a MEMS AO test bed that is already been built and characterized. ${ }^{16}$ The NGAO conceptual design layout for the LOWFS channels is shown in figure 6 . 
The following are the main goals of the prototyping effort:

- Measure read noise from a large format detector (H2RG or InSb) when working as a WFS with selectable pixels and compare to that as obtained from finger testing by looking at a single pixel. Figure 7 shows measured noise from a H2RG detector.

- Ensure that probe arm accuracy and stability conforms to astrometric budget - We will prototype an entire channel including probe arm.

- Determine system performance with varying high order correction level.

- Verify centroiding and spot capturing algorithms.

- Integrate a MEMS DM with a high speed tip-tilt stage at $-20^{\circ} \mathrm{C}$.

\section{CONCLUSION}

A concept to sense low-order wavefront from natural stars is presented using a large format NIR detector. The sensor uses a plate scale of $\sim 80-100$ mas/pixel $(3 \times-4 \times$ the diffraction limit in J-and H-band) for guiding on stars fainter than $M_{J}=16$. The sensor is capable of handling extended objects using a larger box for centroiding. We have also looked at performance of the sensor at varying levels of high order correction. Preliminary tests show that $6 e^{-} s$ RON can be obtained from the detector we have in mind for this purpose when read at $\sim 200$ $\mathrm{Hz}$. The authors plan to prototype the sensor in the lab to test performance and charecterize performance and understand effects that were not modeled. The authors are working closely with the TMT project office and the Herzberg Institute of Astrophysics in choosing Low-order Wavefront sensor architecture for IRIS, a first generation TMT instrument.

\section{ACKNOWLEDGEMENTS}

This material is based in part upon work supported by the National Science Foundation under Grant Number AST-0619922 (P.I.: R. G. Dekany). This work was also funded by W. M. Keck Observatory's Next Generation AO project, SNAP and internal Caltech Optical Observatories funds. The Authors extend thanks to the NGAO and the Palomar Adaptive Optics teams for useful insights.

\section{REFERENCES}

1. Keck Adaptive Optics Note 511 NGAO: System Design Manual, P. Wizinowich et. al., http://www.oir.caltech.edu/twiki_oir/pub/Keck/NGAO/NewKAONs/KAON511_NGAO_SDM_v2.1.pdf

2. Sharpening low-order NGS using patrolling laser guide stars, R. Dekany et. al., SPIE 7015 (2008)

3. Concept for the Keck Next Generation Adaptive Optics System ,D. Gavel et. al. SPIE 7015 (2008)

4. Keck Adaptive Optics Note 551 - Keck Next Generation Adaptive Optics WFS sub-system conceptual study report, V. Velur et al. http://www.oir.caltech.edu/twiki_oir/pub/Keck/NGAO/AOSystemDesign/WFS_design_report_rev5.pdf

5. Modeling low order aberrations in laser guide star adaptive optics systems, R. Clare et. al., Optics Express 15(8).

6. Clare, R., et al., 2006, Adaptive optics sky modeling for extremely large telescopes, Applied Optics, 45, 8964.

7. Lower noise for IR wavefront sensors (based on real noise measurements of 2.5 um H2RG-19), COO talk, R. Smith et. al.

8. Keck Adaptive Optics Note 487 - NGAO trade study report: LOWFS architecture, R. Flicker, V. Velur, http://www.oir.caltech.edu/twiki_oir/pub/Keck/NGAO/WorkProducts/lowfs.pdf

9. Keck Adaptive Optics Note 499 - NGAO System Architecture Definition, R. Dekany et al, http://www.oir.caltech.edu/twiki_oir/pub/Keck/NGAO/NewKAONs/KAON_499_v1.2.pdf

10. Keck Next Generation Adaptive Optics -Science Case Requirements Document, L. McGrath et. al., 
http://www.oir.caltech.edu/twiki_oir/bin/viewfile/Keck/NGAO/NewKAONs?

rev=1; filename=455_NGAO_SCRD_Release2.2_v1.pdf

11. Keck Adaptive Optics Note 492 - Null-modes and quadratic mode tomography error in LGS-based multibeacon tomography AO systems, R. Flicker, C. Neyman, http://www.oir.caltech.edu/twiki_oir/pub/Keck/NGAO/NewKAONs/ftomo.pdf

12. Keck Adaptive Optics Note 429 - NGAO trade study report: LGS asterism geometry and size, R. Flicker, http://www.oir.caltech.edu/twiki_oir/pub/Keck/NGAO/WorkProducts/KA0N_429_LGSasterismTS.pdf

13. Keck Adaptive Optics Note 501 - Keck Next Generation Adaptive Optics Background and Transmission Budgets, A. Bouchez, http://www.oir.caltech.edu/twiki_oir/pub/Keck/NGAO/NewKAONs/KAON501_ngao_bkg_v1-1.pdf

14. Keck Adaptive Optics Note 303-Atmospheric Parameters for Mauna Kea, C. Neyman, http://www.oir.caltech.edu/twiki_oir/pub/Keck/NGAO/NewKAONs/KA0N303v2.pdf

15. Keck Adaptive Optics Note 470 - Keck NGAO sky coverage modeling, R. M. Clare http://www.oir.caltech.edu/twiki_oir/pub/Keck/NGAO/WorkProducts/KAON470.pdf

16. CAMERA: A Compact, Automated, Laser Adaptive Optics System for Small Aperture Telescopes, M. Britton et. al., SPIE 7015 (2008), http://eraserhead.caltech.edu/papers/camera_spie.pdf

17. Fowler A.M., Gatley I., 1990, ApJ Lett. 353, L33 